\title{
Composição química e valor nutricional para grandes herbívoros das folhas e frutos de aninga (Montrichardia linifera, Araceae)
}

\author{
Cristine Bastos do AMARANTE ${ }^{1}$, Regina Celi Sarkis MÜLLER² ${ }^{2}$ Kelly das Graças Fernandes DANTAS³, \\ Cláudio Nahum ALVES ${ }^{4}$, Adolfo Henrique MÜLLER 5 , Dulcidéia da Conceição PALHETA ${ }^{6}$
}

\begin{abstract}
RESUMO
Montrichardia linifera (Araceae), conhecida popularmente como 'aninga', faz parte dos ecossistemas de várzea da Amazônia e da dieta natural de animais como peixe-boi, tartarugas, peixes, búfalo e gado. Com o objetivo de contribuir para o conhecimento químico e valor nutricional da mesma, folhas e frutos de M. linifera foram coletados às margens dos rios Guamá e Maratauíra, no Estado do Pará, Brasil. Em folhas e frutos foram realizadas análises de umidade, resíduo mineral fixo (cinzas), lipídios, proteínas, fibra bruta, concentração de carboidratos e valor calórico. A composição mineral (Ca, Mg, $\mathrm{Cu}, \mathrm{Fe}, \mathrm{Zn}$ e $\mathrm{Mn})$ foi obtida por espectrometria de absorção atômica de chama. Observou-se que tanto as folhas quanto os frutos da aninga, apesar de calóricos (289,75 kcal e 355,12 kcal, respectivamente), possuem baixo valor protéico (0,44\% e 0,24 \%, respectivamente). As concentraçôes de manganês obtidas (folha $=3279,46 \mathrm{mg} \mathrm{kg}^{-1} \mathrm{e}$ fruto $=18151,53 \mathrm{mg} \mathrm{kg}^{-1}$ ) foram consideradas tóxicas, extrapolando o limite máximo tolerável para ruminantes $\left(1000 \mathrm{mg} \mathrm{kg}^{-1}\right)$. A $M$. linifera, tem capacidade de absorver e bioacumular grandes quantidades de $\mathrm{Ca}, \mathrm{Mg}$ e $\mathrm{Mn}$ presentes no solo, o que torna inadequada a sua utilizaçáo exclusiva na alimentação de quelônios, bovinos e bubalinos, havendo necessidade de mais estudos para sua aplicação como parte da ração.
\end{abstract}

PALAVRAS-CHAVE: Nutrição, herbívoro, ruminante, planta aquática

\section{Chemical composition and nutritional value of leaves and fruits of aninga (Montrichardia linifera, Araceae) for large herbivores}

\begin{abstract}
The aninga (Montrichardia linifera, Araceae) is often found in the floodplain ecosystems of the Amazon and is the natural diet of animals such as manatees, turtles, fish, buffalo and cattle. Aiming to contribute to the chemical knowledge and nutritional value of this plant, leaves and fruits of $M$. linifera were collected on the banks of the Guama and Maratauira rivers, Para State, Brazil. We determined the moisture content, ash mineral composition, lipids, protein, fiber, carbohydrate and caloric value of the fruits and leaves. The mineral composition $(\mathrm{Ca}, \mathrm{Mg}, \mathrm{Cu}, \mathrm{Fe}, \mathrm{Zn}$ and $\mathrm{Mn}$ ) was obtained by flame atomic absorption spectrometry. The leaves and the fruits of $M$. linifera had caloric values of $289.75 \mathrm{kcal}$ and $355.12 \mathrm{kcal}$, respectively; and a low protein concentration, $0.44 \%$ for leaves and $-0.24 \%$ for fruits. Manganese concentrations were $3279.46 \mathrm{mg} \mathrm{kg}^{-1}$ for leaves and $18151.53 \mathrm{mg} \mathrm{kg}^{-1-}$ for fruits. These $\mathrm{Mn}$ concentrations are considered toxic, as they exceed the maximum tolerable for the ruminants $\left(1000 \mathrm{mg} \mathrm{kg}^{-1}\right)$. The $M$. linifera has the capacity to absorb and bioaccumulate large amounts of $\mathrm{Ca}, \mathrm{Mg}$ and $\mathrm{Mn}$ in the soil, which makes it inappropriate for exclusive use as food for turtles, cattle and buffaloes, requiring more studies for its application as part of the diet.
\end{abstract}

KEYWORDS: Nutrition, herbivore, ruminant, aquatic plant

\footnotetext{
${ }^{1}$ Museu Paraense Emílio Goeldi, E-mail: cbamarante@museu-goeldi.br

2 Universidade Federal do Pará, E-mail: reginasarkis@hotmail.com

${ }^{3}$ Universidade Federal do Pará, E-mail: kdgfernandes@ufpa.br

${ }^{4}$ Universidade Federal do Pará, E-mail: nahum@ufpa.br

${ }^{5}$ Centro Universitário do Pará,E-mail: muller@cesupa.br

${ }^{6}$ Universidade Federal Rural da Amazônia, E-mail: deia.palheta@ufra.edu.br
} 


\section{INTRODUÇÃO}

Montrichardia linifera (Arruda) Schott, família Araceae, conhecida popularmente como aninga, é uma macrófita aquática anfíbia vastamente distribuída nas várzeas amazônicas e igualmente encontrada em diversos ecossistemas inundáveis como os igapós, margens de rios, furos e igarapés. Em geral, se distribui nas regióes tropicais (Mayo et al.1997), ocorrendo nos Estados brasileiros do Piauí, Rio de Janeiro, sul do país e Suriname (Macedo et al., 2005), porém não há registro de ocorrência no Pantanal Brasileiro. Na literatura há relatos de que suas folhas e frutos servem de alimento aos peixes e quelônios, tais como a tartaruga e o tracajá (Lins, 1994; Portal et al., 2002) e também aos mamíferos de grande porte como o peixe-boi (Lins, 1994), o gado bovino (Furtado, 2002) e o búfalo.

A $M$. linifera também possui tradição etnofarmacológica. A seiva leitosa e urticante do caule dessa espécie é utilizada pelos caboclos ribeirinhos principalmente na cicatrização de cortes profundos (VFH, 2007) e também contra picadas de cobra e ferradas de arraia (Amorozo e Gély, 1998). No entanto, apesar da ocorrência expressiva, estudos químicos envolvendo esta planta ainda são desconhecidos e muito pouco se conhece sobre o seu valor nutritivo. Neste sentido é de relevante importância o estudo da composição química desta espécie, sobretudo a sua avaliação nutricional, pois há a hipótese de que esta planta tenha potencial para compor uma ração regional que poderá ser utilizada tanto na criaçáo de bovinos e bubalinos como na quelonicultura.

Atualmente, a região Amazônica destaca-se na criação de búfalos no Brasil, possuindo $50 \%$ do contingente nacional. Nessa regiáo os bubalinos encontram habitat ideal para o seu desenvolvimento, exercendo papel importante na economia local (Barbosa et al., 2005). O uso da aninga na alimentaçấo de quelônios tem potencialidade, haja vista que quelônios amazônicos, em especial a tartaruga-da-amazônia (Podocnemis expansa, Schweigger, 1812) e o tracajá (Podocnemis unifilis, Troschel, 1848) são espécies liberadas para criação em cativeiro, com altíssimo potencial para exploraçâo zootécnica (Sá et al., 2004).

A análise química foliar é um método muito utilizado na avaliaçấo bromatológica das plantas (Siebeneichler $e t$ al., 2002; Carneiro et al., 2006) e o conhecimento da composiçấo mineral de frutos proporciona subsídios para um programa de nutrição animal (Hiroce et al., 1977). Neste sentido, o objetivo deste trabalho foi conhecer a composição bromatológica das folhas e frutos de $M$. linifera, por meio da determinaçâo dos teores de umidade, resíduo mineral fixo (cinzas), fibra bruta, proteínas, lipídios, carboidratos e os teores de macro e micronutrientes ( $\mathrm{Ca}, \mathrm{Cu}, \mathrm{Fe}, \mathrm{Mg}, \mathrm{Mn}$ e $\mathrm{Zn}$ ) e desta maneira contribuir na avaliação de seu emprego adequado como componente de ração regional, para utilizaçáo na criação de bovinos, bubalinos e quelônios.

\section{MATERIAL E MÉTODOS}

\section{Material vegetal}

Folhas e frutos de Montrichardia linifera foram coletados no período chuvoso. As folhas completamente desenvolvidas com médias de $44 \mathrm{~cm}$ de largura e $52 \mathrm{~cm}$ de comprimento foram coletadas em março, no horário entre 07:30 e 09:00 h, durante a maré baixa, em cinco pontos eqüidistantes ao longo da extensáo da orla do Campus da Universidade Federal do Pará (UFPA), margem direita do Rio Guamá (012'1,3" S; 48²7’29,0” W), em Belém, no estado do Pará. Os frutos foram coletadas em abril, no horário entre 09:00 e 10:00 h, durante a maré alta, em três pontos ao longo do igarapé Furo do Boto, no Rio Maratauíra (0142’05,62” S; 4852'53,06” W), município de Abaetetuba, Pará. As plantas foram identificadas na Coordenaçáo de Botânica do Museu Paraense Emílio Goeldi) e dois exemplares foram depositados neste Herbário (MG 188906 - amostra de Belém; MG 189287- amostra de Abaetetuba). As amostras de folhas e frutos selecionadas foram lavadas com água corrente e depois com água destilada. Parte da amostra foi utilizada para a determinação dos teores de umidade e de cinzas e o restante foi processado para posterior determinação de lipídios, proteínas, carboidratos, fibra bruta e minerais, de acordo com as metodologias descritas a seguir.

\section{Determinação da composição nutricional e mineral}

A umidade e a matéria mineral fixa (cinzas) foram determinadas por gravimetria de acordo com metodologia do Instituto Adolfo Lutz (IAL, 1985). Os lipídios foram determinados por extraçáo em aparelho Soxhlet, utilizando-se éter de petróleo como solvente (IAL, 1985). A torta residual desta extração foi reservada para análise de fibra bruta extraída por hidrólise ácida seguida por hidrólise básica (Cecchi, 2003). O teor de proteínas foi determinado pelo método do biureto. Em uma alíquota de $1 \mathrm{~mL}$ de solução da amostra foram adicionados $4 \mathrm{~mL}$ do reagente biureto e medida a absorbância à 540 nm em espectrofotômetro (CE 1010, Cecil Instruments Ltd, Cambridge, Reino Unido) e os resultados comparados com a curva padrão de albumina bovina (Sigma A-2153, USA), na faixa de 0 a $1 \mathrm{~g} \mathrm{~L}^{-1}$. A fraçáo de carboidratos foi estimada por diferença na composição relativa, isto é, foi representado e calculado por [100 - somatório das demais fraçôes (lipídios, proteínas, fibra bruta e cinzas)] em base seca. O valor calórico foi determinado conforme Furlan et al. (2007), multiplicando-se o teor lipídico por nove e os teores de proteínas e de carboidratos por quatro, sendo expresso em quilocalorias (kcal). Para determinação dos minerais, aproximadamente $0,3 \mathrm{~g}$ de cada amostra foram digeridas com uma mistura de ácido nítrico $\left(14 \mathrm{~mol} \mathrm{~L}^{-1}\right)$ e peróxido 
de hidrogênio (30\% v/v), na proporção de 3:1, em um bloco digestor a $150^{\circ} \mathrm{C}$. Após a digestão, as amostras foram filtradas e transferidas para balóes volumétricos de $50 \mathrm{~mL}$, e em seguida foram aferidos até a marca com água deionizada. Para $\mathrm{Ca}$ e $\mathrm{Mg}$ foi realizada uma nova diluiçáo onde uma alíquota de 0,05 $\mathrm{mL}$ da primeira diluição foi adicionada a $3 \mathrm{~mL}$ de soluçáo 5 $\mathrm{g} \mathrm{L}^{-1}$ de lantânio e $4 \mathrm{~mL}$ de água deionizada. Os teores de $\mathrm{Ca}$, $\mathrm{Cu}, \mathrm{Fe}, \mathrm{Mn}, \mathrm{Mg}$ e $\mathrm{Zn}$ foram determinados nos digeridos por espectrometria de absorção atômica de chama (Varian Spectra AA 220, Mulgrave, Austrália).

\section{RESULTADOS E DISCUSSÃO}

Os valores médios com seus respectivos desvios padrão obtidos, neste trabalho, das folhas e frutos da $M$. linifera (dados a $100 \%$ de matéria seca) referentes ao período chuvoso são apresentados na Tabela 1. Estes valores foram comparados com os resultados obtidos por Portal et al. (2002) e por outros autores que estudaram a composição química de plantas aquáticas na Tabela 2. Cabe aqui mencionar que existe pouca informação sobre a caracterização química e nutricional desta espécie. Os únicos dados encontrados na literatura até o momento são 3,53\% de proteína; 0,59\% de lipídios e $1,32 \%$ de cinzas para amostras secas de caule e fruto de $M$. linifera coletadas às margens dos rios e lagos da regiāo do Pracuúba, Amapá (Portal et al., 2002), no período chuvoso. O teor de umidade encontrado nas folhas (79\%) de M. linifera foi compatível com os valores reportados por outros autores que estudaram macrófitas aquáticas e encontraram elevado teor de umidade nestas plantas, tais como os relatados por Henry-Silva e Camargo (2002) que encontraram valores superiores a 90\% de água nos tecidos vegetais de Eichhornia crassipes (Mart.) Solms, Pistia stratiotes L. e Salvinia molesta DS Mitchell. Corrêa et al. (2003) também destacam o fato de as espécies Egeria densa Planch, Egeria najas Planch e Ceratophyllum demersum L. apresentarem teores de umidade elevados. Os frutos de $M$. linifera também apresentaram um alto teor de umidade $(88,7 \%)$ enquadrando-se na classe dos frutos carnosos e suculentos. O teor de lipídios encontrado nos frutos $(0,88 \%)$ se aproximou do valor relatado por Portal et al. (2002) para os frutos de $M$. linifera que foi de $0,59 \%$. Estes autores também encontraram valor semelhante $(0,51 \%)$ no fruto de Simaba multiflora Adr. Juss. O teor de lipídios obtido das folhas foi maior que nos frutos $(3,7 \%)$ e se enquadrou na faixa de valores encontrados em folhas de outras espécies de plantas aquáticas, tais como 2,7\% em folhas de Anona sp. (Portal et al., 2002), 4,7\% em folhas de E. crassipes, 4,4\% em $P$. stratiotes, 3,8\% em S. molesta (Henry-Silva e Camargo, 2002).

O teor de proteína nos frutos $(0,24 \%)$ foi muito inferior ao valor encontrado por Portal et al. (2002) que foi de 3,53\%. No entanto, estes autores também encontraram baixo teor de proteína nos frutos de S. multiflora $(0,72 \%)$. Silva (2000)
Tabela 1 - Composição nutricional das folhas e frutos da aninga (M. linifera) coletadas no período chuvoso com base na matéria seca (média \pm desvio padrão).

\begin{tabular}{|c|c|c|}
\hline PERÍODO CHUVOSO & FOLHA & FRUTO \\
\hline Umidade (\%) & $79,00 \pm 1,51$ & $88,73 \pm 0,91$ \\
\hline Cinzas (\%) & $6,68 \pm 0,91$ & $7,72 \pm 0,55$ \\
\hline Lipídios (\%) & $3,75 \pm 0,23$ & $0,88 \pm 0,25$ \\
\hline Fibra bruta (\%) & $25,57 \pm 1,21$ & $4,60 \pm 0,54$ \\
\hline Proteína (\%) & $0,44 \pm 0,08$ & $0,24 \pm 0,08$ \\
\hline Carboidratos (\%) & $63,56 \pm 3,94$ & $86,56 \pm 2,33$ \\
\hline $\mathrm{Ca}\left(\mathrm{g} \mathrm{kg}^{-1}\right)$ & $19,15 \pm 0,03$ & $19,67 \pm 0,02$ \\
\hline $\operatorname{Mg}\left(\mathrm{g} \mathrm{kg}^{-1}\right)$ & $5,73 \pm 0,05$ & $2,27 \pm 0,02$ \\
\hline $\mathrm{Fe}\left(\mathrm{mg} \mathrm{kg}^{-1}\right)$ & $70,79 \pm 8,65$ & $35,85 \pm 4,17$ \\
\hline $\mathrm{Cu}\left(\mathrm{mg} \mathrm{kg}^{-1}\right)$ & $7,08 \pm 0,43$ & $5,31 \pm 0,11$ \\
\hline $\mathrm{Zn}\left(\mathrm{mg} \mathrm{kg}^{-1}\right)$ & $272,02 \pm 1,66$ & $159,81 \pm 2,35$ \\
\hline $\operatorname{Mn}\left(\mathrm{mg} \mathrm{kg}^{-1}\right)$ & $3279,46 \pm 22,49$ & $18151,53 \pm 35,12$ \\
\hline Valor calórico (kcal) & 289,75 & 355,12 \\
\hline
\end{tabular}

$\mathrm{Ca}=$ Cálcio; $\mathrm{Mg}=$ Magnésio; $\mathrm{Fe}=$ Ferro, $\mathrm{Cu}=$ cobre; $\mathrm{Zn}=$ Zinco; $\mathrm{Mn}=$ Manganês

também obteve um baixo teor de proteína (em torno de $0,58 \%$ ) no extrato aquoso de sementes frescas de $M$. linifera coletadas em áreas de várzeas dos estuários amazônicos nos municípios de Belém, Barcarena, Abaetetuba, Salvaterra, Cachoeira do Arari e Breves. O teor de proteína das folhas $(0,44 \%)$, neste trabalho, foi próximo ao encontrado na folha, caule e raiz de E. crassipes (0,64\%), por Portal et al. (2002), por outro lado, muito inferior aos valores encontrados por Henry-Silva e Camargo (2002) nas espécies E. crassipes (7,2\%), P. stratiotes $(8,8 \%)$ e S. molesta $(8,7 \%)$, no entanto há que se considerar que estes últimos valores foram obtidos da biomassa total do material vegetal. Também foi observada diferença no resultado do teor de cinzas 6,68\% (folhas) e 7,72\% (frutos) sete vezes superior ao valor publicado por Portal et al. (2002) para a $M$. linifera, que foi de $1,32 \%$, evidenciando maior concentraçáo de minerais presentes no solo nos locais de coleta deste trabalho.

Henry-Silva e Camargo (2002) estudando a composição bromatológica de macrófitas aquáticas flutuantes também observaram variaçôes intraespecíficas nos conteúdos de nutrientes dos tecidos vegetais e associaram essas variações à influência das diferentes condiçôes tróficas dos ambientes aquáticos. O elevado percentual de cinzas presente nas folhas e nos frutos de $M$. linifera provavelmente está associado à interface química entre a planta e o ambiente circundante, considerando-se que se trata de uma planta aquática de área de várzea, que sofre alagamentos diários e periódicos (Salomão $e t$ al., 2007) favorecendo o acúmulo de sedimentos no solo. Os 
Tabela 2 - Valores comparativos obtidos de folhas de M. linifera neste trabalho com os obtidos por outros autores em plantas aquáticas

\begin{tabular}{|c|c|c|c|c|c|c|c|c|c|c|c|c|c|}
\hline \multirow[t]{2}{*}{ Espécie } & \multirow{2}{*}{$\begin{array}{l}\text { Parte da } \\
\text { planta }\end{array}$} & \multicolumn{5}{|c|}{$\begin{array}{l}\text { Composição Relativa } \\
(\%)\end{array}$} & \multicolumn{2}{|c|}{$\begin{array}{c}\text { Macronutrientes } \\
\left(\mathrm{g} \mathrm{kg}^{-1}\right)\end{array}$} & \multicolumn{4}{|c|}{$\begin{array}{l}\text { Micronutrientes } \\
\left(\mathrm{mg} \mathrm{kg}^{-1}\right)\end{array}$} & \multirow[t]{2}{*}{ Autores } \\
\hline & & Umid & $\operatorname{Cinz}$ & Lip & $\mathrm{Fib}$ & Prot & $\mathrm{Ca}$ & $\mathrm{Mg}$ & $\mathrm{Fe}$ & $\mathrm{Cu}$ & $\mathrm{Zn}$ & $\mathrm{Mn}$ & \\
\hline M. linifera* & Folha & 79,00 & 6,68 & 3,75 & 25,57 & 0,44 & 19,15 & 5,73 & 70,79 & 7,08 & 272,02 & 3279,46 & Neste trabalho \\
\hline M. arborescens* & Folha & - & - & - & - & - & 18,50 & 4,62 & 1150,00 & - & 259,00 & - & Busetti et al (2006) \\
\hline $\begin{array}{l}\text { E. crassipes } \\
\text { P. stratiotes* } \\
\text { S. molesta }\end{array}$ & $\begin{array}{l}\text { Biomassa } \\
\text { total }\end{array}$ & $>90$ & - & $\begin{array}{l}4,70 \\
4,40 \\
3,80\end{array}$ & - & $\begin{array}{l}7,2 \\
8,8 \\
8,7\end{array}$ & - & - & - & - & - & - & $\begin{array}{l}\text { Henry-Silva e } \\
\text { Camargo (2002) }\end{array}$ \\
\hline $\begin{array}{l}\text { E. crassipes } \\
\text { P. stratiotes* }\end{array}$ & $\begin{array}{c}\text { Biomassa } \\
\text { total }\end{array}$ & - & - & $\begin{array}{l}4,73 \\
4,44\end{array}$ & $\begin{array}{l}17,09 \\
18,95\end{array}$ & - & $\begin{array}{l}15,10 \\
10,90\end{array}$ & $\begin{array}{l}3,92 \\
2,15\end{array}$ & $\begin{array}{l}5425,00 \\
1391,67\end{array}$ & $\begin{array}{c}25,83 \\
9,17\end{array}$ & $\begin{array}{l}81,83 \\
29,83\end{array}$ & $\begin{array}{l}1233,33 \\
2145,83\end{array}$ & $\begin{array}{l}\text { Henry-Silva e } \\
\text { Camargo (2006) }\end{array}$ \\
\hline E. crassipes & Folha & - & - & - & - & 0,64 & 30,20 & - & - & - & - & - & \multirow{2}{*}{ Portal et al. (2002) } \\
\hline Anona sp. & Folha & - & 0,81 & 2,69 & 22,00 & 1,97 & 14,40 & 3,20 & - & - & - & - & \\
\hline $\begin{array}{l}\text { E. crassipes } \\
\text { P. stratiotes* } \\
\text { S. auriculata }\end{array}$ & $\begin{array}{c}\text { Biomassa } \\
\text { total }\end{array}$ & - & - & - & - & - & $\begin{array}{c}11,55 \\
22,35 \\
9,18\end{array}$ & $\begin{array}{l}2,93 \\
4,50 \\
2,48\end{array}$ & $\begin{array}{l}4671,33 \\
4050,00 \\
3206,00\end{array}$ & $\begin{array}{c}9,75 \\
8,58 \\
11,14\end{array}$ & $\begin{array}{l}187,00 \\
233,27 \\
250,33\end{array}$ & $\begin{array}{l}1254,00 \\
1319,00 \\
1491,00\end{array}$ & $\begin{array}{l}\text { Martins et al. } \\
\quad(2003)\end{array}$ \\
\hline $\begin{array}{l}\text { E. densa } \\
\text { E. najas } \\
\text { C. demersum }\end{array}$ & $\begin{array}{c}\text { Biomassa } \\
\text { total }\end{array}$ & $\begin{array}{l}- \\
- \\
-\end{array}$ & $\begin{array}{l}- \\
-\end{array}$ & $\begin{array}{l}- \\
- \\
-\end{array}$ & $\begin{array}{l}- \\
- \\
-\end{array}$ & $\begin{array}{l}- \\
- \\
-\end{array}$ & $\begin{array}{l}17,30 \\
16,50 \\
11,80\end{array}$ & $\begin{array}{l}3,60 \\
7,60 \\
9,80\end{array}$ & $\begin{array}{l}2154,90 \\
2958,50 \\
3526,20\end{array}$ & $\begin{array}{l}5,90 \\
5,70 \\
4,60\end{array}$ & $\begin{array}{l}102,90 \\
130,50 \\
149,00\end{array}$ & $\begin{array}{l}- \\
-\end{array}$ & $\begin{array}{l}\text { Corrêa,Velini e } \\
\text { Arruda (2003) }\end{array}$ \\
\hline M. linifera* & Fruto & 88,73 & 7,72 & 0,88 & 4,60 & 0,24 & 19,67 & 2,27 & 35,85 & 5,31 & 159,81 & 18151,5 & Neste trabalho \\
\hline M. linifera* & Fruto & - & 1,32 & 0,59 & - & 3,53 & - & - & - & - & - & - & \multirow{4}{*}{ Portal et al. (2002) } \\
\hline S. multiflora & Fruto & - & - & 0,51 & - & 0,72 & - & - & - & - & - & - & \\
\hline M. sprucena & Fruto & - & - & - & 6,80 & - & - & - & - & - & - & - & \\
\hline 0. aquática & Fruto & - & - & & 7,60 & - & - & - & - & - & - & - & \\
\hline M. linifera* & Sementes & - & - & - & - & 0,58 & - & - & - & - & - & - & Silva (2000) \\
\hline
\end{tabular}

Legenda: *Espécies da família Araceae; Umid=umidade; Cinz=cinzas; Lip=lipídios; Fib=Fibra bruta; Prot=proteína; Ca=Cálcio, Mg=magnésio; Fe=ferro; Cu=cobre; $\mathrm{Zn}=$ zinco; $\mathrm{Mn}=$ manganês.

ambientes de várzea agem como purificadores de águas não tratadas, retendo grande quantidade de nutrientes e metais pesados, funcionando como verdadeiros filtros (Ramos et al., 2003) biogeoquímicos (Eschrique et al., 2003), logo, essas substâncias vão se acumulando no solo e consequentemente são absorvidas pela planta. Rubio et al. (2004) afirmam que as plantas aquáticas são sorventes naturais de substâncias químicas e que o seu uso no controle da poluição ambiental ainda é pouco explorado, já que elas tem grande capacidade de reter metais pesados, óleos e outros poluentes orgânicos.

O teor de fibras encontrado nas folhas $(25,57 \%$, com base na matéria seca) foi próximo à maioria dos valores encontrados em outras espécies por Portal et al. (2002), sendo que nove espécies apresentaram teores elevados $(>30 \%)$ de fibra bruta. Por outro lado, o teor de fibras encontrado nos frutos $(4,6 \%)$ ficou bem abaixo deste valor. No entanto, duas outras espécies estudadas por estes autores apresentaram frutos com teores próximos aos encontrados neste trabalho, 6,8\% em Marlierea spruceana O. Berg e 7,6\% em Ouratea aquatica (Kunth) Engl.

Os altos teores de cinzas relatados neste estudo (Tabela 2), indicam que folhas e frutos apresentaram elevados teores de minerais. De fato, as concentraçôes de cálcio encontradas nas folhas $\left(19,1 \mathrm{~g} \mathrm{~kg}^{-1}\right)$ e nos frutos $\left(19,7 \mathrm{~g} \mathrm{~kg}^{-1}\right)$ de $M$. linifera foram superiores aos valores encontrados por Portal et al. (2002) em seis espécies, assemelhando-se principalmente à
Genipa sp. $\left(19 \mathrm{~g} \mathrm{~kg}^{-1}\right)$. As concentraçóes de magnésio nas folhas $\left(5,73 \mathrm{~g} \mathrm{~kg}^{-1}\right)$ e nos frutos $\left(2,27 \mathrm{~g} \mathrm{~kg}^{-1}\right)$ de $M$. linifera também foram compatíveis com oito espécies estudadas Portal et al. (2002).

Busetti et al. (2006) encontraram valores similares de Ca $\left(18,5 \mathrm{~g} \mathrm{~kg}^{-1}\right)$ e $\mathrm{Mg}\left(4,6 \mathrm{~g} \mathrm{~kg}^{-1}\right)$ em folhas de Montrichardia arborescens (L.) Schott coletadas no Furo do Maguari, em Belém-PA. De um modo geral, em termos de macronutrientes, pode-se constatar que as folhas de $M$. linifera analisadas neste trabalho apresentaram teores de $\mathrm{Ca}$ e $\mathrm{Mg}$ em proporçóes semelhantes aos dos valores reportados por outros autores para plantas aquáticas (Portal et al., 2002; Henry-Silva e Camargo, 2002; Martins et al., 2003; Corrêa et al., 2003; Busseti et al., 2006; Henry-Silva e Camargo, 2006). Em relação aos micronutrientes, os teores de $\mathrm{Cu}, \mathrm{Zn}$ e Mn também oscilaram na faixa de valores reportados por estes mesmos autores em outras espécies, sendo o teor de Fe a única exceção, ficando muito abaixo dos valores relatados até mesmo para $M$. arborescens. No entanto, ainda na Tabela 2 observa-se que a $M$. arborescens foi a espécie que apresentou o menor teor de Fe quando comparada às demais espécies.

Em se tratando da aplicação de $M$. linifera na alimentação animal, as altas concentraçôes de $\mathrm{Ca}$ e $\mathrm{Mg}$ encontradas nas folhas e frutos desta espécie sugerem que os mesmos poderiam ser fornecidos aos quelônios na forma de suplemento alimentar, 
porém a escassez de dados a respeito das necessidades mínimas requeridas e as quantidades máximas toleradas pelos quelônios (Sá et al., 2004) exigem cautela na sua utilização em raçóes, principalmente por causa dos elevados teores de manganês encontrados em ambos. Com esses dados pode-se concluir que as folhas e frutos de $M$. linifera são de baixo valor protéico para sua utilizaçáo isolada na quelonicultura.

Os resultados da composição mineral encontrados nas folhas e frutos da aninga são comparados, na Tabela 3 , com os valores das necessidades mínimas e quantidades máximas toleráveis admitidas para bovinos de corte segundo o NRC (2007). Os resultados encontrados fora desses limites estáo destacados em negrito.

Tabela 3 - Teores de minerais (média \pm desvio padrão) encontrados nas folhas e frutos da aninga (Montrichardia linifera) comparados com os valores das quantidades mínimas requeridas e máximas toleráveis segundo o NRC.

\begin{tabular}{lcccc}
\hline & \multicolumn{2}{c}{ ANINGA (M. linifera) } & \multicolumn{2}{c}{ NRC* (2007) } \\
\cline { 2 - 5 } & FOLHA & FRUT0 & $\begin{array}{c}\text { Mínimo } \\
\text { Requerido }\end{array}$ & $\begin{array}{c}\text { Máximo } \\
\text { Tolerável }\end{array}$ \\
\hline Ca (\%) & $1,92 \pm 0,003$ & $1,97 \pm 0,002$ & 0,18 & -------- \\
Mg (\%) & $\mathbf{0 , 5 7 \pm 0 , 0 0 5}$ & $0,23 \pm 0,002$ & 0,10 & 0,40 \\
Fe (mg kg-1) & $70,79 \pm 8,65$ & $\mathbf{3 5 , 8 5} \pm 4,17$ & 50,00 & 1000,00 \\
Cu $\left(\mathrm{mg} \mathrm{kg}^{-1}\right)$ & $\mathbf{7 , 0 8} \pm 0,43$ & $\mathbf{5 , 3 1} \pm 0,11$ & 10,00 & 100,00 \\
Zn (mg kg-1) & $272,02 \pm 1,66$ & $159,81 \pm 2,35$ & 30,00 & 500,00 \\
Mn $\left(\mathrm{mg} \mathrm{kg}^{-1}\right)$ & $\mathbf{3 2 7 9 , 4 6} \pm 22,49$ & $\mathbf{1 8 1 5 1 , 5 3 \pm 3 5 , 1 2}$ & 20,00 & 1000,00 \\
\hline
\end{tabular}

$\mathrm{Ca}=$ Cálcio; $\mathrm{Mg}=$ Magnésio; $\mathrm{Fe}=$ Ferro, $\mathrm{Cu}=$ cobre; $\mathrm{Zn}=\mathrm{Zinco} ; \mathrm{Mn}=$ Manganês . ${ }^{*} \mathrm{NRC}=$ National Research Council

As folhas apresentaram teores de $\mathrm{Ca}$, Fe e $\mathrm{Zn}$ acima das exigências mínimas para a nutrição de gado. No entanto, os teores obtidos para magnésio e manganês foram acima do nível de tolerância admitido para bovinos, sendo que a concentração de manganês obtida foi três vezes maior que o máximo tolerável nas folhas e dezoito vezes no fruto, ultrapassando a faixa considerada tóxica para a maioria das criaçóes. Os elevados teores de manganês encontrados tanto nas folhas quanto nos frutos, podem ser justificados pelo fato de que o solo permanece muito tempo inundado e, como a concentração de manganês na solução do solo aumenta após a submersão do solo (Mattar, 2002), decorrente do processo de redução, ocorrerá maior disponibilidade de manganês no solo (Iwata, 1975) e maior absorção pela planta (Abreu et al., 2006). Além disso, amostras de solo coletadas numa área próxima à área de estudo desta pesquisa, mostraram valores de $\mathrm{pH}<5$ indicando solos muito ácidos (Lopes et al., 2006; Abreu et al., 2007). Condiçôes de $\mathrm{pH}$ ácido favorecem o acúmulo de concentraçôes tóxicas de manganês, em virtude do aumento da solubilidade em pH 5,0 (Foy, 1973).

Nesse sentido, apesar de os valores nutricionais de produtos vegetais serem influenciados principalmente por seu perfil genético, acredita-se que os baixos teores de proteína encontrados nas folhas e frutos da aninga podem ser também ser influenciados pela acidez do solo, visto que Abreu et al. (2007) observaram na margem do Rio Guamá que a concentração de nitrogênio foi significativamente menor no período de maior inundaçáo, em todas as profundidades de amostragens e áreas estudadas. Em solos ácidos e anaeróbios com $\mathrm{pH}<5,5$, a taxa de nitrificação é muito baixa, o que resulta em acumulo de amônia $\left(\mathrm{NH}_{3}\right)$, que pode ser perdida por volatilização. Além disso, o nitrogênio na forma nítrica pode ser perdido por denitrificação, reduzindo assim sua concentração no solo. As folhas apresentaram deficiência de cobre assim como os frutos. Estes valores baixos de cobre obtidos podem estar relacionados com o excesso de manganês. Veloso et al. (1995) observaram em Piper nigrum L. uma relação inversa entre o $\mathrm{Cu}$ e o $\mathrm{Mn}$. Outro fator a ser considerado é o fato de que os solos paraenses, por natureza, têm baixo teor de cobre. Estudos realizados por Sousa et al., (2007) no município de Melgaço, Pará, mostraram que o teor de cobre no solo variou de baixo a médio, resultado compatível com o estudo de Barbosa et al. (2007) que cita o Pará como um dos Estados que apresentam deficiência deste mineral em bovinos e ovinos. Isto se deve, provavelmente, à baixa disponibilidade deste metal nos solos paraenses, e por consequência, nas forragens. No entanto, ainda na Tabela 2, observa-se que a maioria das espécies aquáticas estudadas por outros autores (Corrêa, et al., 2003) apresentam baixos teores de $\mathrm{Cu}$, independente do local de coleta.

Os frutos apresentaram teor de Fe abaixo do mínimo requerido $\left(35,85 \mathrm{mg} \mathrm{kg}^{-1}\right)$ e as folhas, apesar de conterem teores de Fe dentro da faixa estabelecida $\left(70,79 \mathrm{mg} \mathrm{kg}^{-1}\right)$, ainda assim, apresentaram valores relativamente baixos, próximos ao mínimo requerido $\left(50,00 \mathrm{mg} \mathrm{kg}^{-1}\right)$. A relação $\mathrm{Mn} / \mathrm{Fe}$ nos tecidos de plantas tem um interesse peculiar, tendo em vista que alguns autores associam a toxidez do Mn à deficiência de Fe. Por exemplo, Lee (1972) associou esta toxidez a uma razáo $\mathrm{Mn} / \mathrm{Fe}$ de $18 \mathrm{em}$ batateiras (Solanum tuberosum L. var. 'Netted Gem') e Veloso et al. (1995) associaram uma relaçấo $\mathrm{Mn} / \mathrm{Fe}$ de 22 a sintomas severos de toxidez em folhas de pimenteiras (Piper nigrum L.). De fato, Veloso et al. (1995) sugerem que as quantidades contidas de alguns nutrientes, tais como $\mathrm{N}, \mathrm{P}, \mathrm{Cu}$, $\mathrm{Fe}$, e Zn, na parte aérea das plantas, podem ser afetadas pela presença de manganês na solução do meio para crescimento das plantas, a partir de $20 \mathrm{mg} \mathrm{L}^{-1}$, onde foram observados sintomas nas folhas das pimenteiras.

As folhas das plantas de aninga coletadas à margem direita do Rio Guamá apresentaram cloroses marginais e entre as nervuras, com pequenos pontos necróticos no limbo, provavelmente indicativos de toxidez devido ao acúmulo de manganês nas folhas. $\mathrm{O}$ manganês, quando em excesso na solução do solo, promove reduçóes nas quantidades absorvidas 
de ferro pelas plantas (Foy et al., 1978) justificando os resultados obtidos com a $M$. linifera, neste trabalho.

Os desequilíbrios dos minerais na dieta animal podem ocorrer tanto pela deficiência como pelo excesso (Ribeiro, 1997). Barbosa et al. (2005) descreveram achados clínicos e epidemiológicos das enfermidades que acometem búfalos na Amazônia, principalmente no Estado Pará, e durante o período de 1997 a 2005 foram diagnosticadas várias enfermidades, porém entre as que mais chamaram a atençáo foram as deficiências minerais, principalmente de fósforo e cobre. Estes autores relataram que em búfalos na Ilha do Marajó e nordeste do Pará tem-se observado animais com presença de pêlos de coloraçáo amarronzada (cor de ferrugem), sendo que este sintoma tem sido atribuído à deficiência de cobre. Ainda segundo estes autores, a alteração congênita é outra enfermidade que se destaca nos búfalos da Amazônia, em particular a falta de coordenaçáo dos movimentos (ataxia) do bezerro búfalo. Essa enfermidade acomete o sistema nervoso e vem sendo diagnosticada em bubalinos recém-nascidos nos Municípios paraenses de Castanhal, Ipixuna e Santa Izabel. Os animais portadores desta enfermidade apresentaram crescimento retardado e dificuldade de acompanhar as mães durante o pastejo. Estes dados chamam a atenção para a importância de mais estudos com relaçáo à absorção do Mn pelas plantas das áreas inundáveis, que fazem parte da alimentação natural dos búfalos, para verificar se há relaçôes entre a ocorrência de ataxia e as concentraçôes de Mn presente na dieta destes animais.

Desta forma, os resultados para a $M$. linifera (folhas e frutos) sugerem uma espécie considerada de baixo valor proteico também para bubalinos e bovinos, pois teores de proteína inferiores a $7 \%$ são limitantes à produçáo animal (Minson, 1990). Além disso, devido aos elevados teores de manganês, pode ser considerada uma planta tóxica para estes animais. Falta ainda se determinar como produtos derivados de animais que consomem altas concentraçóes de Mn (p.exe: carnes e produtos lácteos) podem influenciar na saúde de humanos.

Foi concluído que a utilização das folhas e frutos da aninga (Montrichardia linifera, Araceae) na alimentação de quelônios, bovinos e bubalinos é imprópria para a criação destes animais por apresentarem, em sua composição química, teores tóxicos de magnésio e manganês além de um baixo valor protéico.

\section{AGRADECIMENTOS}

Os autores agradecem à Dra. Alba Lins, da Coordenação de Botânica do Museu Paraense Emílio Goeldi (MPEG) pela identificação da espécie, informaçóes e orientaçôes. Ao técnico do Laboratório de Análises Minerais da Universidade Federal Rural da Amazônia (UFRA), Ricardo Oliveira e Silva, pelo auxílio prestado nas análises espectrométricas. À Fundação de
Amparo à Pesquisa do Estado do Pará (FAPESPA) pela bolsa de doutorado concedida à primeira autora.

\section{BIBLIOGRAFIA CITADA}

Abreu, E.M.A.de, Fernandes, A.R.; Ruivo, M.L.P. 2007. Temporary and vertical variation of chemical attributes of a gleysol of the Guamá River cultivated with canaranas. Revista Brasileira de Ciência do Solo, 31: 277-285 (in Portuguese, with abstract in English).

Abreu, E.M.A. de; Fernandes, A.R.; Martins, A.R.A.; Rodrigues, T.E. 2006. Forage production and nutritive value of forage species under pasture conditions in lowland soils of the Guamá River. Acta Amazonica, 36:11-18 (in Portuguese, with abstract in English).

Amorozo, M. C. M.; Gély, A. L. 1998. Use of medicinal plants by the caboclos of the Lower Amazon. Barcarena, PA, Brazil. Boletim do Museu Paraense Emílio Goeldi, Sér. Botânica 4: 47-131 (in Portuguese, with abstract in English).

Barbosa, F.A.; Graça, D.S.; Silva Júnior, F.V. da. 2007. Mineral deficiencies of cattle in tropical pastures. Diagnosis and Supplementation. Portal Agronomia: Artigos Científicos. (www. agronomia.com.br/conteudo/artigos/artigos_deficiencias_ minerais.htm). Acesso: 20/07/08 (in Portuguese).

Barbosa, J.D.; Oliveira, C.M.C.; Duarte, M.C.; Silveira, J.A.S. 2005. Disease of Buffalo in the Amazon. In: II Simpósio Mineiro de Buiatria, Belo Horizonte. (www.ivis.org/proceedings/ abmg/2005/pdf11.pdf?LA=7). Acesso: 26/08/2008 (in Portuguese).

Busetti, E. P. C.; Ruivo, M.L.; Sales, M. E. ; Berrêdo, J.F. 2006. Distribution of nutrients in soil and vegetation in a floodplainestuary system, mangrove in Belém, p. 105-133. In: Castro, E. (Org). Belém of water and islands. Cejup, Belém, Pará (in Portuguese).

Carneiro, C.; Reissmann, C.B.; Marques, R. 2006. Comparation of methods for chemical analysis of $\mathrm{K}, \mathrm{Ca}, \mathrm{Mg}$ and $\mathrm{Al}$, in maté tea tree (Ilex paraguariensis St. Hil.) Cerne, 12: 113-122 (in Portuguese, with abstract in English).

Cecchi, H.M. 2003. Theoretical and practical fundamentals in analysis of food. Universidade Estadual de Campinas, Campinas, São Paulo, Brazil. 208 pp (in Portuguese).

Corrêa, M.R.; Velini, E.D.; Arruda, D.P. 2003. Chemical and bromatological composition of Egeria densa, Egeria najas and Ceratophyllum demersum. Planta Daninha, 21:7-13 (in Portuguese, with abstract in English).

Eschrique, S. A.; Abreu, M. W. M.; Alves, M. A. M.; Santos, M. L. S. 2003. Preliminary studies on the factors that control the concentration of nutrients (phosphate and silicate) in the estuary of the Bispo Beach - Mosqueiro/PA. In: IX Congresso Brasileiro de Geoquímica. Anais, Belém, Pará, Brasil, p.492-494 (in Portuguese, with abstract in English).

Foy, C.D.; Chaney, R.L.; White, M.C. 1978. The physiology of metal toxicity in plants. Annual Review of Plant Physiology, 29: 511-566.

Foy, C.D. 1973. Manganese and plants, p. 51-76. In:__ Manganese. National Academy of Sciences, Washington, D.C. 
Furlan, E.F.; Galvão, J.A.; Salán, E.O.; Yokoyama, V.A.; Oetterer, M. 2007. Physicochemical stability and market of mussels (Perna perna) cultivated in Ubatuba - SP, Brasil.Ciência e Tecnologia de Alimentos, 27: 516-523 (in Portuguese, with abstract in English).

Furtado, M.B. 2002. Contribution to the study of the extraction process and the physical characterization of fiber from Aninga (Montrichardia linifera Schott). Dissertação de Mestrado, Universidade Federal do Pará, Belém, Pará. 48 pp (in Portuguese).

Henry-Silva, G.G.; Camarago, A.F.M. 2002. Nutritive value of free-floating aquatic macrophytes (Eichhornia crassipes, Pistia stratiotes and Salvinia molesta), used in aquaculture waste treatment. Acta Scientiarum, 24: 519-526 (in Portuguese, with abstract in English).

Henry-Silva, G.G.; Camargo, A.F.M. 2006. Chemical Composition of Floating Aquatic Macrophytes Used to Treat of Aquaculture Wastewater. Planta Daninha, 24: 21-28 (in Portuguese, with abstract in English).

Hiroce, R.; Carvalho, A.M.; Bataglia, O.C.; Furlani, P.R.; Furlani, A.M.; Santos, R.R.; Pariquera-Açu, E.E.; Gallo, J.R. 1977. Mineral composition of tropical fruits at harvest. Bragantia, Revista Cientifica do Instituto Agrônomico do Estado de São Paulo, 36: 155-164 (in Portuguese).

IAL (Instituto Adolpho Lutz). 1985. Chemical and physical methods for food analysis. Analytical Standards, São Paulo, Brazil, 317 pp (in Portuguese).

Iwata, T. 1975. Studies on the occurrence of tomebagare a newly found physiological disease of Rice and its peventive measures. Fukui: Fukui Agriultural Experiment Station, 6: 1-66.

Lee, C.R. 1972. Interrelationships of aluminium and manganese on the potato plant. Agronomy Journal, 64: 546-549.

Lins, A. L. F. A. 1994. Morphological and anatomical aspects of roots of genus Montrichardia Crüger. (Araceae). Dissertaçáo de Mestrado, Universidade Federal do Rio Grande do Sul, Porto Alegre, Rio Grande do Sul. 91 pp (in Portuguese).

Lopes, E.L.N.; Fernandes, A.R.; Grimaldi, C.; Ruivo, M.L.P.; Rodrigues, T.E.; Sarrazin, M. 2006. Chemical characteristics of a Gleysoil under different soil use systems along the Guamá river banks in Belém, Pará. Boletim do Museu Paraense Emílio Goeldi sér. Ciências Naturais, 1: 127-137 (in Portuguese, with abstract in English).

Macedo, E. G.; Santos Filho, B. G.; Potiguara, R. C. V.; Santos, D. S. B. 2005. Leaf anatomy and architecture of Montrichardia linifera (Arruda) Schott (Aracea) a specie from Amazon floodplain. Boletim do Museu Paraense Emílio Goeldi sér. Ciências Naturais, 1: 19-43 (in Portuguese, with abstract in English).

Martins, D.; Costa, N.V.; Terra, M.A.; Marchi, S.R.; Velini, E.D. 2003. Chemical characterization of aquatic plants assessed in Salto Grande reservoir (Americana-SP, Brazil). Planta Daninha, 21: 21-25 (in Portuguese, with abstract in English).

Mattar, R.M.V.C.; Vieira, I.S.; Silva, G.R. da. 2002. Effect of flooding on $\mathrm{pH}$ values and in the solubility of phosphorus, sodium, iron and manganese of a "low humic gley" soil from the Amazon Basin of Brazil. Revista de Ciências Agrárias, 37: 113-157 (in Portuguese, with abstract in English).
Mayo, S.J.; Bogner, J.; Boyce, P.C. 1997. The Genera of Araceae. Royal Botanic Gardens, Kew, United Kingdom. 370 pp.

Minson, D.J. 1990. Forage in ruminant nutrition. Academic Press, London, 483pp.

NRC (National Research Council). 2007. Nutrient requeriments of beef cattle. National Academy of Sciences, Washington. (http:// www.aces.edu/pubs/docs/A/ANR-0060/ANR-0060.pdf). Acesso: 08/08/2008.

Portal, R.R.; Lima, M.A.S.; Luz, V.L.F.; Bataus, Y.S.L. 2002. Vegetables species used as food by Podocnemis unifilis, Troschel 1948 (Reptilia: Testudinae,Pelomedusidae) in the Pracuúba Region, State of Amapá/Brazil. Ciência Animal Brasileira 3: 1119 (in Portuguese, with abstract in English).

Ramos, D. G. B.; Polivanov, H.; Mendonça Filho, J.G.; Rezende, A. S. Geochemistry of heavy metals in soils of the mangrove ecosystem - Duque de Caxias (RJ). 2003. In: IX Congresso Brasileiro de Geoquímica, Anais, Belém, Pará, Brasil, p.470-472 (in Portuguese, with abstract in English).

Ribeiro, S.D.A. 1997. Rational rearing of goat. Nobel, São Paulo, Brazil. 318 pp (in Portuguese).

Rubio, J.; Schneider, I. A. H.; Ribeiro, T.; Costa, C. A.; Kallfez, C. A. 2004. Aquatic plants: natural sorbents. Ciência Hoje, 35: 68-71 (in Portuguese).

Sá, V.A.; Quintanilha, L.C.; Freneau, G.E.; Luz, V.L.F.; Borja, A.L.R.; Silva, P.C. 2004. Body growth of one-month Giant Amazonian Turtle (Podocnemis expansa) fed isocaloric diet with different levels of crude protein concentration. Revista Brasileira de Zootecnia 33: 2351-2358 (in Portuguese, with abstract in English).

Siebeneichler, S.C.; Monnerat, P.H.; Carvalho, A.J.C.; Silva, J.A. 2002. Mineral composition of the leaf in pineapple: effect of the part of the analysed leaf. Revista Brasileira de Fruticultura, 24: 194-198 (in Portuguese, with abstract in English).

Salomão, R. P.; Terezo, E. F. M.; Rosa, N. A.; Ferreira, L. V.; Matos, A. H.; Adams, M.; Amaral, D. D.; Morais, K. A. C. 2007. Forest management in the floodplain: characterization, restrictions and opportunities for adoption, p.11-138. In: Salomão, R. P.; Terezo, E. F. M.; Jardim, M. A. G. (Orgs.). Forest management in the floodplain: opportunities and challenges. Museu Paraense Emílio Goeldi, Belém, Pará, Brazil (in Portuguese).

Silva, C. S. 2000. Purification, partial characterization and properties of a lectin from Montrichardia linifera (Arruda) Schott. Dissertaçáo de Mestrado, Universidade Federal do Rio Grande do Sul, Porto Alegre, Rio Grande do Sul. 104 pp (in Portuguese).

Sousa, A.F.; Amaral, I.G., Baía, P.P.S.; Alves, R.F.T. 2007. Evaluation of physico-chemical properties of soil in the National Forest of Caxiuaná, Melgaço-Pará. In: 59a Reunião Anual da SBPC. Resumos. (www.servicos.sbpcnet.org.br/sbpc/sbpc/59ra/senior/ livroeletronico/resumos/R3578-1.html). Acesso: 03/08/2008.

Veloso, C.A.C.; Muraoka, T.; Malavolta, E.; Carvalho, J.G. 1995. Effect of manganese on mineral nutrition and growth of black pepper (Piper nigrum, L.). Scientia Agricola, 52: 376-383 (in Portuguese, with abstract in English). 
ACTA

AMAZONICA

Composição química e valor nutricional para grandes herbívoros

das folhas e frutos de aninga (Montrichardia linifera, Araceae)

VFH (Virtual Field Herbarium). 2007. (http://herbaria.plants.

ox.ac.uk/VFH/image/index.php?item=116\&taxonomy=698>).

Acesso: 27/01/2007.

Recebido em 20/12/2009

Aceito em 16/04/2010 